\title{
Simulated synchrotron emission from pulsar wind nebulae
}

\author{
L. Del Zanna ${ }^{1}$, D. Volpi ${ }^{1}$, E. Amato ${ }^{2}$, and N. Bucciantini ${ }^{3}$ \\ 1 Dipartimento di Astronomia e Scienza dello Spazio, Università di Firenze, Largo E. Fermi 2, 50125 Firenze, Italy \\ e-mail: 1dz@arcetri.astro.it \\ 2 INAF - Osservatorio Astrofisico di Arcetri, Largo E. Fermi 5, 50125 Firenze, Italy \\ 3 Astronomy Department, University of California at Berkeley, 601 Campbell Hall, Berkeley, CA 94720-3411, USA
}

Received 14 January 2006 / Accepted 21 March 2006

\section{ABSTRACT}

\begin{abstract}
Aims. A complete set of diagnostic tools aimed at producing synthetic synchrotron emissivity, polarization, and spectral index maps from relativistic MHD simulations is presented. As a first application we consider here the case of the emission from Pulsar Wind Nebulae (PWNe).

Methods. The proposed method is based on the addition, on top of the basic set of MHD equations, of an extra equation describing the evolution of the maximum energy of the emitting particles. This equation takes into account adiabatic and synchrotron losses along streamlines for the distribution of emitting particles and its formulation is such that it is easily implemented in any numerical scheme for relativistic MHD.

Results. Application to the axisymmetric simulations of PWNe, analogous to those described by Del Zanna et al. (2004), allows direct comparison between the numerical results and observations of the inner structure of the Crab Nebula, and similar objects, in the optical and X-ray bands. We are able to match most of the observed features typical of PWNe, like the equatorial torus and the polar jets, with velocities in the correct range, as well as finer emission details, like arcs, rings and the bright knot, that turn out to arise mainly from Doppler boosting effects. Spectral properties appear to be well reproduced too: detailed spectral index maps are produced for the first time and show softening towards the PWN outer borders, whereas spectral breaks appear in integrated spectra. The emission details are found to strongly depend on both the average wind magnetization, here $\sigma_{\text {eff }} \approx 0.02$, and on the magnetic field shape.

Conclusions. Our method, in spite of its simplicity, provides a realistic modeling of synchrotron emission properties, and twodimensional axisymmetric relativistic MHD simulations appear to be well suited to explain the main observational features of PWNe.
\end{abstract}

Key words. radiation mechanisms: non-thermal - Magnetohydrodynamics (MHD) - relativity - pulsars: general ISM: supernova remnants - ISM: individual objects: Crab Nebula

\section{Introduction}

Non-thermal radiation, and synchrotron emission from relativistic particles in magnetized plasmas in particular, is a ubiquitous signature in high energy astrophysics. Strong shocks in relativistic flows are believed to be the natural site for the acceleration of particles up to relativistic velocities. High energy electrons and positrons, then, efficiently emit synchrotron radiation while spiraling around magnetic field lines. For optically thin sources, the emission spectrum, typically a power law, is related to the particles' distribution function at injection through well known transport and radiation physics. However, the connection may be not easy to establish quantitatively in the presence of multidimensional flow patterns, making it difficult to extract from observations direct information on the most basic physics of the sources.

Environments where synchrotron emission is encountered span from extragalactic sources, like cluster halos or Active Galactic Nuclei (AGNs), for example jets and lobes in radioloud quasars, to the bulge and disk of our Galaxy, and galactic sources like Supernova Remnants (SNRs). Precisely to a subclass of the latter belongs one of the most studied astrophysical sources: the Crab Nebula. The Crab Nebula is the prototype of the so-called Pulsar Wind Nebulae (PWNe), or plerions, and it is the object where synchrotron radiation was first identified (Shklovsky 1953). The emission from PWNe displays all the characteristic properties of synchrotron radiation from relativistic particles: a continuous, very broad-band spectrum, extending from radio to X-rays and beyond (though Inverse Compton typically dominates at gamma-ray frequencies) and a high degree of linear polarization; spectral indices, defined by $F_{v} \propto v^{-\alpha}$ where $F_{v}$ is the net flux, range between $0 \lesssim \alpha \lesssim 1.2$ and in a given source usually steepen with increasing frequency.

While the theoretical modeling of the emitted radiation soon reached a high level of sophistication (e.g. Kennel \& Coroniti $1984 b$, for PWNe), what has been lacking until very recently is an appropriate modeling of the fluid and magnetic structure of the relativistic plasma responsible for the emission. In the last few years, however, there has been an increasingly rapid growth in accuracy and robustness of shock-capturing numerical schemes for relativistic hydrodynamics and MHD (see Martí \& Müller 2003; and Font 2003, for periodically updated reviews), so that nowadays relativistic simulations are becoming a standard investigation tool for plasma astrophysics. Among the others, we mention here the works on special relativistic MHD by Komissarov (1999) and Del Zanna et al. (2003), with the latter putting forward a simpler and yet powerful alternative to characteristics-based algorithms. Based on these techniques, conservative shock-capturing schemes for MHD in General Relativity (GRMHD) have also been constructed (e.g. Gammie et al. 2003; Komissarov 2005; Antón et al. 2006), 
especially for studies of the accretion flows around Kerr black holes or extraction of gravitational energy and subsequent jet acceleration. Recently, coupling of conservative schemes for GRMHD with solvers for the Einstein equations has been achieved too (Duez et al. 2005), for studies of binary neutron star mergers, core collapse in supernovae, black hole formation and gamma-ray burst generation.

The goal of the present paper is to provide the numerical community with a simple computational tool that can be used to compute the brightness, polarization and spectrum of the synchrotron light associated with the plasma dynamics resulting from a given MHD simulation. The method proposed is quite general and can be applied to any scheme for relativistic MHD (not necessarily in conservative form, either in full 3-D or under particular symmetries) and it just requires the numerical solution of an additional evolution equation, as we will discuss later. As a first application of our techniques we study here in detail the emission properties of PWNe, as they can be computed based on the recent axisymmetric model by Del Zanna et al. (2004).

The interest of the scientific community for PWNe has recently received considerable impulse from the X-ray observations made by Chandra and XMM-Newton, showing that the Crab Nebula and other PWNe all present complex but similar inner structures: a higher emission torus in what is thought to be the equatorial plane of pulsar rotation, typically with brighter features like arcs or rings, a central knot, and one or two opposite jets along the polar axis, apparently originating from very close to the pulsar position (Hester et al. 1995, for Hubble and ROSAT earlier observations; Weisskopf et al. 2000; Hester et al. 2002; Helfand et al. 2001; Pavlov et al. 2001; Gotthelf \& Wang 2000; Gaensler et al. 2001, 2002; Lu et al. 2002; Camilo et al. 2004; Slane et al. 2004; Romani et al. 2005). This scenario is generally referred to as the jet-torus structure.

While the presence of an equatorial torus of enhanced emission can be explained by applying the standard 1-D relativistic MHD solutions (Kennel \& Coroniti 1984a; Emmering \& Chevalier 1987) and assuming that the pulsar wind has a higher equatorial energy flux (Bogovalov \& Khangoulian 2002), the origin of the polar jets has puzzled plasma physicists until recently, given the known difficulties in obtaining selfcollimation via hoop stresses by the toroidal field in an ultrarelativistic MHD outflow (Lyubarsky \& Eichler 2001 and references therein). However, an axisymmetric energy flux enhanced at the equator produces an oblate termination shock with a cusplike shape at the poles. Moreover, if hoop stresses are at work in the mildly relativistic post-shock flow, jet collimation may occur on the axis at these cusps, thus giving the impression of an origin inside the wind itself (Lyubarsky 2002). This scenario has been recently confirmed by relativistic MHD numerical simulations (Komissarov \& Lyubarsky 2003, 2004; Del Zanna et al. 2004), which all show that the termination shock really assumes the predicted shape, polar jets with velocities in the observed range $0.5-0.8 c$ are formed (provided the wind magnetization is high enough (approximately 1\%), and the complex flow patterns which arise near the termination shock are responsible for most of the observed brighter features, basically just due to Doppler boosting effects (Komissarov \& Lyubarsky 2004).

Compared to the quality of the simulation results for the flow structure, synchrotron emission modeling has been so far rather crude. In the only attempt so far at computing synchrotron surface brightness maps on top of the flow structure obtained from 2-D MHD simulations (Komissarov \& Lyubarsky 2004) radiative losses were approximated by an exponentially decaying factor, with a spatially constant cooling time depending on the frequency of observation and an average magnetic field. A power law distribution function for the emitting particles, normalized to the local thermal pressure to take into account variation of specific volume, was assumed in the emissivity. A more sophisticated method was proposed by Shibata et al. (2003): here several particle energies are evolved independently in order to reconstruct the initial distribution function at any location and time. However, this method is suitable only for nearly steady-state solutions where the individual paths (the fluid streamlines) can be unambiguously followed. The method proposed here is a step forward concerning the application to time-dependent numerical schemes: emitting particles are continuously injected at the termination shock and then the full particles' energy equation is integrated in time. However, differently from the the above cited paper, just the maximum energy attainable is evolved, and it is taken as the upper cut-off in the local power law distribution function that enters the emission coefficient.

Other than just synchrotron surface brightness, we also show here how to build polarization maps, namely maps of Stokes parameters, degree of linear polarization, and position angle. Such data are available for the Crab Nebula in radio (Wilson 1972; Velusamy 1985) and optical (Schmidt et al. 1979; Hickson \& van der Berg 1990; Michel et al. 1991), though high resolution data are still missing. X-ray polarization maps are not available yet. Synthetic maps have been reported in a preliminary work (Bucciantini et al. 2005b), where optical maps have been produced for one test case, here we discuss the dependence on the model parameters. Finally, our method allows us to produce detailed spectral maps, which can be compared to optical observations (e.g. Véron-Cetty \& Woltjer 1993) and to Chandra and XMM-Newton X-ray data (Weisskopf et al. 2000; Willingale et al. 2001; Mori et al. 2004). Synthetic spectral index maps can provide us with an additional diagnostic tool for the inner magnetic structure of PWNe, since the spectral softening due to synchrotron losses depends on the local magnetic field. To our knowledge, this is the first time synthetic synchrotron spectral index maps based on data from numerical simulations have been produced.

The paper is structured as follows: the proposed method is described in Sect. 2, the main PWN model assumptions are summarized in Sect. 3, numerical results and synthetic synchrotron maps are shown in Sect. 4, where also a comparison with observations is attempted. Finally, Sect. 5 is dedicated to the conclusions.

\section{Synthetic synchrotron emission recipes}

In the present section a method for producing synthetic synchrotron surface brightness and polarization maps, based on the results of numerical simulations, will be derived and discussed. The proposed method takes into account both adiabatic and synchrotron losses in the evolution of the emitting particles advected by the nebular flow, and it can be easily implemented in any numerical scheme for relativistic MHD equations. The only assumption made here is that the code provides the variables $\rho$ (proper mass density), $\boldsymbol{v}$ (flow bulk velocity), $p$ (proper thermal pressure), and $\boldsymbol{B}$ (magnetic field) as functions of space, with or without special symmetries reducing the dimensionality of the system, and time. Lorentz transformations leading to relativistic corrections in the emission properties, like Doppler shift and boosting or polarization angle swing, will also be fully considered. Therefore, in the following subsections, different notations will be employed where ambiguities may occur: primed quantities will refer to the reference frame comoving with the 
fluid, whereas standard notation will indicate quantities in the observer's frame.

\subsection{Distribution function evolution}

The observed spectra in astrophysical sources of non-thermal radiation can be often approximated by power laws, with varying spectral index $\alpha$ in different frequency regimes. It is therefore natural to model the emitting particles' (typically electrons and positrons) distribution function at injection locations as a combination of power laws as well. Let then $\epsilon_{0}$ be the particle's energy (in units of the rest mass energy $m c^{2}$ ) and consider a distribution function which is isotropic in momentum and power law in energy, within a given energy range:

$f_{0}\left(\epsilon_{0}\right)=\frac{A}{4 \pi} \epsilon_{0}^{-(2 \alpha+1)}, \quad \epsilon_{0}^{\min } \leq \epsilon_{0} \leq \epsilon_{0}^{\max }$,

where from now on quantities labeled with the 0 index will refer to the injection region. The constant of proportionality

$A=K_{n} n_{0}=K_{p} \frac{p_{0}}{m c^{2}}$

can be determined by the definitions of the proper number density $n_{0}$ and thermal pressure $p_{0}$ in terms of $f_{0}\left(\epsilon_{0}\right)$, provided $\epsilon_{0}^{\max } \gg \epsilon_{0}^{\min }$ (see Kennel \& Coroniti 1984b). If $\alpha>1 / 2$, as it is the case in the following, integration over momentum space and energy gives

$\left\{\begin{array}{l}K_{n}=2 \alpha\left(\epsilon_{0}^{\min }\right)^{2 \alpha} \\ K_{p}=3(2 \alpha-1)\left(\epsilon_{0}^{\min }\right)^{2 \alpha-1},\end{array}\right.$

and from the above definitions we also find

$\epsilon_{0}^{\min }=3\left(\frac{2 \alpha-1}{2 \alpha}\right) \frac{p_{0}}{n_{0} m c^{2}}$,

that can be determined by the Rankine-Hugoniot relations if the injection location is a shock $\left(\epsilon_{0}^{\mathrm{min}}\right.$ is of order of the upstream wind Lorentz factor, $\sim 10^{6}$ for PWNe).

Time evolution of the single energies along post-shock streamlines is governed by adiabatic and radiative losses. If $\mathrm{d} / \mathrm{d} t^{\prime}=\gamma(\partial / \partial t+\boldsymbol{v} \cdot \nabla)$ is the total time derivative in the comoving frame, the equation for $\epsilon$ is

$$
\frac{\mathrm{d}}{\mathrm{d} t^{\prime}} \ln \epsilon=\frac{\mathrm{d}}{\mathrm{d} t^{\prime}} \ln n^{1 / 3}+\frac{1}{\epsilon}\left(\frac{\mathrm{d} \epsilon}{\mathrm{d} t^{\prime}}\right)_{\text {sync }}
$$

where synchrotron losses are

$$
\left(\frac{\mathrm{d} \epsilon}{\mathrm{d} t^{\prime}}\right)_{\mathrm{sync}}=-\frac{4 e^{4}}{9 m^{3} c^{5}} B^{\prime 2} \epsilon^{2}
$$

in which we have averaged over all possible pitch angles with the local magnetic field that a particle may experience during its lifetime. Based on the evolution of the single energies, we now seek an equation for the evolution of the distribution function $f(\epsilon)$ along streamlines. This problem was solved, for a stationary radial flow, by Kennel \& Coroniti (1984b), extended to the multi-dimensional case by Begelman \& Li (1992), and first applied to the results of a time-dependent code by Bucciantini et al. (2005a). From the integration of Eq. (5) along streamlines it is possible to write

$$
\frac{n^{1 / 3}}{\epsilon}-\frac{n_{0}^{1 / 3}}{\epsilon_{0}}=\frac{n^{1 / 3}}{\epsilon_{\infty}}
$$

where $\epsilon_{\infty}$ takes into account the integrated synchrotron losses and corresponds to the remaining energy of a particle with energy $\epsilon_{0} \rightarrow \infty$ at the injection location, that is to say the local maximum energy attainable. By using the above relation it is possible to express $\epsilon$ as a function of $\epsilon_{0}$ and $\epsilon_{\infty}$, which can be easily calculated for a stationary flow everywhere in space. From the conservation of the particles' number $f(\epsilon) \mathrm{d} \epsilon / n=$ $f_{0}\left(\epsilon_{0}\right) \mathrm{d} \epsilon_{0} / n_{0}$ along streamlines we are finally able to derive the distribution function as

$f(\epsilon)=\frac{K_{p}}{4 \pi} \frac{p}{m c^{2}} \epsilon^{-(2 \alpha+1)}\left[\left(\frac{p}{p_{0}}\right)^{1 / 4}\left(1-\frac{\epsilon}{\epsilon_{\infty}}\right)\right]^{2 \alpha-1}, \epsilon<\epsilon_{\infty}$,

where we have used adiabaticity of the relativistically hot plasma: $p \sim n^{4 / 3}$.

The method we propose in the time-dependent case, provided changes in the global structure occur on timescales not shorter than the typical transport times, is to evolve also the variable $\epsilon_{\infty}$ by adding an extra equation to the relativistic MHD code. For a shock-capturing scheme the required conservative form of Eq. (5) is found by combining it with the continuity equation for $\rho=n m$ :

$$
\frac{\partial}{\partial t}(\gamma \rho \mathcal{E})+\nabla \cdot(\gamma \rho \mathcal{E} \boldsymbol{v})=\frac{\rho \mathcal{E}}{\epsilon_{\infty}}\left(\frac{\mathrm{d} \epsilon_{\infty}}{\mathrm{d} t^{\prime}}\right)_{\mathrm{sync}}
$$

with $\mathcal{E}=\epsilon_{\infty} / \rho^{1 / 3}$ and $\epsilon_{\infty}$ being a large enough number at injection, so as to mimic the theoretical behavior over the largest possible domain (differences due to $\epsilon_{0}<\infty$ should be confined to the close vicinities of the injection location, see Kennel \& Coroniti 1984b).

Once we have $\epsilon_{\infty}$ as a function of time and space, we can model the effects of adiabatic and synchrotron losses on the distribution function by applying Eq. (8). For ease of implementation in a numerical code, however, a further approximation is finally needed. Since $n_{0}$ and $p_{0}$ are difficult to trace back starting from the position along the local streamline, in the multidimensional case one should also assume that their influence is negligible. This occurs when two conditions are met: the dependency on the term $\left(p / p_{0}\right)^{1 / 4}$ is weak and so is that on quantities involving the temperature $\sim p_{0} / n_{0}$ at injection. Both conditions are easily satisfied for $2 \alpha-1 \gtrsim 0$, so that $K_{n}$ and $K_{p}$ are approximately constant.

\subsection{The emission coefficient}

Consider an ultra-relativistic electron (or positron) with normalized energy $\epsilon$, which spirals around the local magnetic field $\boldsymbol{B}^{\prime}$. Its synchrotron spectral power (per unit frequency) is given by

$\mathcal{P}\left(v^{\prime}, \epsilon\right)=\frac{2 e^{4}}{3 m^{2} c^{3}} B_{\perp}^{\prime 2} \epsilon^{2} \mathcal{S}\left(v^{\prime}, v_{\mathrm{c}}^{\prime}\right)$

where $B_{\perp}^{\prime}$ is the field component normal to the particle's velocity, the spectral density is

$\mathcal{S}\left(v^{\prime}, v_{\mathrm{c}}^{\prime}\right)=\frac{9 \sqrt{3}}{8 \pi v_{\mathrm{c}}^{\prime}} F\left(\frac{v^{\prime}}{v_{\mathrm{c}}^{\prime}}\right)$,

and the function $F(x)$ (see Rybicki \& Lightman 1979, for its properties) is such that emission peaks around the critical frequency

$v_{\mathrm{c}}^{\prime}(\epsilon)=\frac{3 e}{4 \pi m c} B_{\perp}^{\prime} \epsilon^{2}$ 
The local emission coefficient $j_{v}^{\prime}$ (radiated power per unit frequency, volume and solid angle), for given observation frequency $v^{\prime}$ and direction $\boldsymbol{n}^{\prime}$, is obtained by integrating the product of Eqs. (10) and (8) over the whole range of local particle energies:

$j_{v}^{\prime}\left(v^{\prime}, \boldsymbol{n}^{\prime}\right)=\int \mathcal{P}\left(v^{\prime}, \epsilon\right) f(\epsilon) \mathrm{d} \epsilon$.

Note that, since radiation from ultra-relativistic particles is strongly beamed in their instantaneous direction of motion (within a cone of angle $1 / \epsilon \ll 1$ ), only those particles with pitch angle coinciding with the angle between $\boldsymbol{B}^{\prime}$ and $\boldsymbol{n}^{\prime}$ (thus $\left.B_{\perp}^{\prime}=\left|\boldsymbol{B}^{\prime} \times \boldsymbol{n}^{\prime}\right|\right)$ contribute to the emission along the line of sight in Eq. (13).

The above integral can be calculated by replacing the energy with the variable $x=v^{\prime} / v_{\mathrm{c}}^{\prime} \propto \epsilon^{-2}$. In order to take advantage of the known properties of the function $F(x)$ we further simplify Eq. (8) to a standard power law form

$f(\epsilon)=\frac{K_{p}}{4 \pi} \frac{p}{m c^{2}} \epsilon^{-(2 \alpha+1)}, \quad \epsilon<\epsilon_{\infty}$,

in which we have assumed that $K_{p}$ is constant, the dependency on the term $\left(p / p_{0}\right)^{1 / 4}$ is weak, and synchrotron burn-off can be modeled with a sharp cut-off at $\epsilon_{\infty}$. This last additional assumption is again a good approximation provided $2 \alpha-1 \gtrsim 0$. The result is

$j_{v}^{\prime}\left(v^{\prime}, \boldsymbol{n}^{\prime}\right)=C p\left|\boldsymbol{B}^{\prime} \times \boldsymbol{n}^{\prime}\right|^{\alpha+1} v^{\prime-\alpha}$,

where all spatially independent terms have been gathered in the constant

$C=\frac{\sqrt{3}}{16 \pi} \frac{\alpha+5 / 3}{\alpha+1} \Gamma\left(\frac{\alpha+5 / 3}{2}\right) \Gamma\left(\frac{\alpha+1 / 3}{2}\right) \frac{e^{3}}{m^{2} c^{4}}\left(\frac{3 e}{2 \pi m c}\right)^{\alpha} K_{p}$,

in which the property $\Gamma(\xi+1)=\xi \Gamma(\xi)$ of the Euler gamma function $\Gamma(\xi)$ has been used.

In order to obtain the emissivity in the observer's fixed frame of reference, relativistic corrections must be taken into account. It is well known that both the frequency and the emission coefficient itself transform via the Doppler boosting factor

$D=\frac{1}{\gamma(1-\boldsymbol{\beta} \cdot \boldsymbol{n})}$,

as $v=D v^{\prime}$ and $j_{v}=D^{2} j_{v}^{\prime}$, where $\beta=\boldsymbol{v} / c$. The resulting emission coefficient is finally written as

$j_{v}(v, \boldsymbol{n})= \begin{cases}C p\left|\boldsymbol{B}^{\prime} \times \boldsymbol{n}^{\prime}\right|^{\alpha+1} D^{\alpha+2} v^{-\alpha}, & v_{\infty} \geq v \\ 0, & v_{\infty}<v .\end{cases}$

The cut-off frequency for synchrotron burn-off is

$v_{\infty} \equiv D v_{\mathrm{c}}^{\prime}\left(\epsilon_{\infty}\right)=D \frac{3 e}{4 \pi m c}\left|\boldsymbol{B}^{\prime} \times \boldsymbol{n}^{\prime}\right| \epsilon_{\infty}^{2}$,

where Doppler shift has been considered and where $\epsilon_{\infty}$ is provided directly by the numerical scheme via integration of Eq. (9).

The terms $B^{\prime}$ in Eq. (6), needed to evolve $\epsilon_{\infty}$, and $\left|\boldsymbol{B}^{\prime} \times \boldsymbol{n}^{\prime}\right|$ in Eqs. (18), (19), are defined through quantities in the comoving frame. Application of the composition rule for relativistic velocities to the observer direction versor $\boldsymbol{n}$ yields

$\boldsymbol{n}^{\prime}=D\left[\boldsymbol{n}+\left(\frac{\gamma^{2}}{\gamma+1} \boldsymbol{\beta} \cdot \boldsymbol{n}-\gamma\right) \boldsymbol{\beta}\right]$ while Lorentz transformations of Maxwell's equations in the (ideal) MHD case $\boldsymbol{E}+\boldsymbol{\beta} \times \boldsymbol{B}=0$ provide

$\boldsymbol{B}^{\prime}=\frac{1}{\gamma}\left[\boldsymbol{B}+\frac{\gamma^{2}}{\gamma+1}(\boldsymbol{\beta} \cdot \boldsymbol{B}) \boldsymbol{\beta}\right]$.

After some straightforward algebra, the required quantities can be written as

$B^{\prime}=\frac{1}{\gamma} \sqrt{B^{2}+\gamma^{2}(\boldsymbol{\beta} \cdot \boldsymbol{B})^{2}}$,

and

$\left|\boldsymbol{B}^{\prime} \times \boldsymbol{n}^{\prime}\right|=\frac{1}{\gamma} \sqrt{B^{2}-D^{2}(\boldsymbol{B} \cdot \boldsymbol{n})^{2}+2 \gamma D(\boldsymbol{B} \cdot \boldsymbol{n})(\boldsymbol{\beta} \cdot \boldsymbol{B})}$,

in which everything on the right-hand-side is now measured in the observer's frame.

\subsection{Surface brightness and polarization maps}

To compare the synthetic synchrotron emission obtained by numerical simulations with real images, the first step certainly requires the production of surface brightness maps. Let us consider a Cartesian observer's reference frame in which $X$ lies along the line of sight $\boldsymbol{n}$ and $Y$ and $Z$ are in the plane of the sky (say with $Z$ indicating the North). By switching from the local coordinate system, which may present special symmetries, to the observer's system $(X, Y, Z)$ (taking into account the angles of inclination of the emitting object), surface brightness (or specific intensity) maps are obtained as

$I_{v}(v, Y, Z)=\int_{-\infty}^{\infty} j_{v}(v, X, Y, Z) \mathrm{d} X$

A powerful additional diagnostic tool may be provided by polarization measurements, where available. Synchrotron emission from relativistic particles is known to be linearly polarized, with a high degree of polarization, and Stokes parameters $U_{v}$ and $Q_{v}$ $\left(V_{v}=0\right)$ can be calculated from numerical simulations by measuring the local polarization position angle $\chi$, that is the angle of the emitted electric field vector $\boldsymbol{e}$ in the plane of the sky, measured clockwise from the $Z$ axis. Synthetic maps of the Stokes parameters are thus built as

$Q_{v}(v, Y, Z)=\frac{\alpha+1}{\alpha+5 / 3} \int_{-\infty}^{\infty} j_{v}(v, X, Y, Z) \cos 2 \chi \mathrm{d} X$

$U_{v}(v, Y, Z)=\frac{\alpha+1}{\alpha+5 / 3} \int_{-\infty}^{\infty} j_{v}(v, X, Y, Z) \sin 2 \chi \mathrm{d} X$

where the additional factor comes from the intrinsic properties of synchrotron emission (see Rybicki \& Lightman 1979). Related to the Stokes parameters above is the degree of (linear) polarization, or polarization fraction, defined as

$\Pi_{v}=\frac{\sqrt{Q_{v}^{2}+U_{v}^{2}}}{I_{v}}$

In order to calculate $\chi$, relativistic effects like position angle swing must be taken into account (Blandford \& Königl 1979; Björnsson 1982; Lyutikov et al. 2003). In the comoving frame, the emitted electric field of the linearly polarized radiation is normal to both the local magnetic field $\boldsymbol{B}^{\prime}$ and the line of sight $\boldsymbol{n}^{\prime}$, thus $\boldsymbol{e}^{\prime} \propto \boldsymbol{n}^{\prime} \times \boldsymbol{B}^{\prime}$, and the radiated magnetic field is therefore 
$\boldsymbol{b}^{\prime}=\boldsymbol{n}^{\prime} \times \boldsymbol{e}^{\prime}$. Lorentz transformations give the electric field in the observer frame as

$\boldsymbol{e}=\gamma\left[\boldsymbol{e}^{\prime}-\frac{\gamma}{\gamma+1}\left(\boldsymbol{e}^{\prime} \cdot \boldsymbol{\beta}\right) \boldsymbol{\beta}-\boldsymbol{\beta} \times \boldsymbol{b}^{\prime}\right]$,

and by using Eqs. (20) and (21) we can express the required electromagnetic fields in terms of quantities defined in the observer frame. After some calculations, the simple formula derived by Lyutikov et al. (2003) is retrieved:

$\boldsymbol{e} \propto \boldsymbol{n} \times \boldsymbol{q}, \quad \boldsymbol{q}=\boldsymbol{B}+\boldsymbol{n} \times(\boldsymbol{\beta} \times \boldsymbol{B})$.

Notice that $\boldsymbol{e}$ lies in the plane of the sky, as expected, and that $\boldsymbol{q}$ correctly reduces to $\boldsymbol{B}$ for non-relativistic fluid bulk motions. We are now ready to derive the position angle $\chi$ in terms of the components of $\boldsymbol{q}$ in the plane of the sky, which are

$q_{Y}=\left(1-\beta_{X}\right) B_{Y}+\beta_{Y} B_{X}, \quad q_{Z}=\left(1-\beta_{X}\right) B_{Z}+\beta_{Z} B_{X}$.

The functions of the position angle appearing in Eqs. (25), (26) may be written in terms of these as:

$\cos 2 \chi=\frac{q_{Y}^{2}-q_{Z}^{2}}{q_{Y}^{2}+q_{Z}^{2}}, \quad \sin 2 \chi=-\frac{2 q_{Y} q_{Z}}{q_{Y}^{2}+q_{Z}^{2}}$.

\subsection{Spectral index maps and integrated spectra}

In order to complete our set of diagnostic tools based on the synthetic synchrotron emission obtained from numerical simulations, we now turn to spectral properties. An interesting possibility is provided by maps of spectral index. This quantity is defined as

$\alpha_{v}\left(v_{1}, v_{2}, Y, Z\right)=-\frac{\log \left[I_{v}\left(v_{2}, Y, Z\right) / I_{\nu}\left(v_{1}, Y, Z\right)\right]}{\log \left(v_{2} / v_{1}\right)}$,

for any given interval in frequency $\left[v_{1}, v_{2}\right]$. In general, due to synchrotron radiative losses, this quantity is expected to increase, thus the spectrum is expected to steepen, when moving away from the source of ultra-relativistic particles (spectral softening).

Finally, integrated spectra (or net fluxes) are obtained by averaging the specific intensity over the solid angle covered by the source in the plane of the sky, which may be calculated as

$F_{v}(v)=\frac{1}{d^{2}} \iint I_{v}(v, Y, Z) \mathrm{d} Y \mathrm{~d} Z$,

where $d$ is the distance of the astronomical object under consideration.

Notice that both the above quantities related to spectral features have a meaning only if synchrotron burn-off is modeled in some way by the numerical simulation.

\section{Pulsar wind model and initial settings}

As anticipated in the Introduction, let us now apply our set of diagnostic tools to the modeling of the inner structure of PWNe. Axisymmetric 2-D simulations of the interaction of an ultra-relativistic magnetized pulsar wind with supernova ejecta expanding in the static interstellar medium were described in Del Zanna et al. (2004), to which the reader is referred for all the modeling details and for the results. Here we will calculate the synchrotron emission from the results of new simulations, obtained by using very similar settings. The numerical code employed is described in Del Zanna \& Bucciantini (2002),
Del Zanna et al. (2003), and Londrillo \& Del Zanna (2004). It is a shock-capturing code solving the ideal relativistic MHD equations in conservative form, to which Eq. (9) has been added. Note that the effects of synchrotron radiative losses on the fluid total energy evolution are not taken into account. Radiative losses would become important if the particles radiated away a considerable fraction of the pulsar energy input to the nebula. However, the radiated power is estimated to be of order $10 \%$ of the instantaneous pulsar input in the case of the Crab Nebula, and generally much smaller than this in the case of other PWNe, so the condition of adiabaticity should generally be considered as a good approximation.

Let us briefly summarize the initial conditions and the pulsar wind model in particular. The numerical box is made up of 400 cells in the radial direction, between $r=0.1$ ly and $r=10$ ly (a logarithmic grid with 200 cells per decade is used to enhance resolution in the inner region), and 100 cells in the latitudinal direction, with polar angle $\theta$ defined in the first quadrant only (symmetry conditions are applied at the equator $\theta=\pi / 2$ ). Within an arbitrary radius of 0.2 ly we impose initially the wind conditions, while the expanding ejecta, with linearly increasing radial velocity, occupy the region up to $1 \mathrm{ly}$. The (constant) wind luminosity is taken to be $L_{0}=5 \times 10^{38} \mathrm{erg} \mathrm{s}^{-1}$, the energy of the supernova explosion turned into kinetic energy of the ejecta $E_{\mathrm{ej}}=10^{51} \mathrm{erg}$, and the total mass of the ejected material $M_{\mathrm{ej}}=3 M_{\odot}$. For similar settings and a description on the (1-D) evolution of the PWN / SNR system see van der Swaluw et al. (2001) and Bucciantini et al. (2003). The chosen values were seen to provide an overall evolution compatible with that of the Crab Nebula. Furthermore, the magnetic field $\boldsymbol{B}$ is assumed to retain just the toroidal component, as in Kennel \& Coroniti (1984a), while the velocity $v$ is assumed to be always poloidal, so that some of the relativistic corrections in Sect. 2.2 are simplified since $\boldsymbol{\beta} \cdot \boldsymbol{B}=0$.

The latitudinal dependence of the wind energy flux is provided by the following choice for the wind bulk Lorentz factor

$\gamma(\theta)=\gamma_{0}\left[\alpha_{0}+\left(1-\alpha_{0}\right) \sin ^{2} \theta\right]$

where $\gamma_{0}=100$ is the equatorial value and $\alpha_{0}=0.1$ the anisotropy parameter. Velocity is assumed to be in the radial direction alone at $t=0$. The residual magnetic field at large distances from the pulsar's light cylinder is thought to be mainly toroidal with a dependence $B \sim \sin \theta / r$ (split monopole models: Michel 1973) inside the wind. By prescribing such a field and an isotropic mass flux, the energy flux for a cold ultra-relativistic wind may be then written as

$F(r, \theta)=F_{0}\left(\frac{r_{0}}{r}\right)^{2}\left[\alpha_{0}+\left(1-\alpha_{0}+\sigma\right) \sin ^{2} \theta\right]$

where $\sigma$ is the wind magnetization parameter, namely the ratio of magnetic to kinetic energy fluxes computed at the equator (Kennel \& Coroniti 1984a), and $F_{0}$ is the equatorial kinetic energy flux at the reference radius $r_{0}$, to be derived in terms of $L_{0}$, $\alpha_{0}$, and $\sigma$ by integrating Eq. (35) over a spherical surface of radius $r$.

The value of $\sigma$ is typically expected to be a small number in order to match the predicted and observed nebular emission (Kennel \& Coroniti 1984b: $\sigma=3 \times 10^{-3}$ for the Crab Nebula), thus one is left with the problem of how to convert a Poynting flux dominated outflow near the pulsar to a kinetically dominated wind before the termination shock (the so-called sigma paradox) (see e.g. Arons 2004). One of the solutions proposed invokes the presence a striped wind region around the equator, due to 
the inclination of the pulsar's magnetosphere with respect to the rotation axis, where dissipation in the resulting current sheets may form a neutral (unmagnetized) equatorial region (Coroniti 1990). We model here such a situation by prescribing the wind toroidal field as

$B(r, \theta)=B_{0}\left(\frac{r_{0}}{r}\right) \sin \theta \tanh \left[b\left(\frac{\pi}{2}-\theta\right)\right]$,

where we set $B_{0}=\sqrt{4 \pi \sigma F_{0} / c}$ and where the last factor mimics the conversion of magnetic to kinetic energy in the striped wind. The parameter $b$ controls the angular size of the zone of lower magnetization: a large $b$ indicates a narrow striped wind region, and the ideal limit is recovered by letting $b \rightarrow \infty$. Here we assume that Eq. (35) still holds even for a finite $b$. Finally, the density $\rho$, decreasing as $r^{-2}$, can be derived from the above equations and will depend on $\sigma$ and $b$ too, since it is supposed to account also for the conversion of magnetic to kinetic energy in the striped wind region (note that the mass flux is actually isotropic only in the ideal limit $b \rightarrow \infty$ ).

As our main goal here is the comparison with observations of the Crab Nebula, the temporal evolution of the PWN/SNR interaction will not be followed in any detail. On the contrary, we will focus our attention on the synchrotron emission properties of the simulated PWN at a time corresponding to the age of the $\mathrm{Crab}, t \approx 1000 \mathrm{yr}$, which is still in the free expansion phase (Bucciantini et al. 2003). As shown by Del Zanna et al. (2004), equatorial flows and polar jets with velocities in the range of the observed values are found for an effective magnetization of the wind plasma $\sigma_{\text {eff }} \gtrsim 0.01$, where $\sigma_{\text {eff }}$ is obtained by averaging over polar angle (the magnetization in the wind region does not depend on $r$ ). Thus, here we will consider two different runs, $\mathrm{A}$ and $\mathrm{B}$, with approximately the same $\sigma_{\text {eff }} \approx 0.02$ and very different magnetic fields:

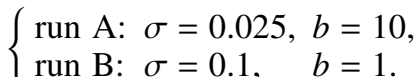

The wind magnetization $B^{2} / 4 \pi \rho c^{2} \gamma^{2}$ for the two runs as a function of the polar angle $\theta$ is shown in Fig. 1. Notice the difference in the striped wind region width around the equator: run A refers to a nearly ideal case, as those discussed by Del Zanna et al. (2004), while in run B the striped wind region is very wide.

\section{Simulation results}

\subsection{Flow structure}

Before studying the synchrotron radiation maps, let us briefly comment on the flow structure and related properties for the two different runs, focusing on the quantities that are relevant for computing the emission. In Fig. 2 we show the velocity field (velocity magnitude and streamlines), the magnetization $B^{2} / 4 \pi w \gamma^{2}$ ( $w=\rho c^{2}+4 p$ is the enthalpy, dominated by the kinetic component in the pulsar wind and by the thermal one inside the nebula), and the maximum particle energy $\epsilon_{\infty}$. The latter is evolved according to Eq. (9) and its value at injection is $10^{9}$. Initialization of $\epsilon_{\infty}$ occurs, like for all other quantities, in the wind region, so that the value of $10^{9}$ is further enhanced at the crossing of the termination shock, due to the conservation of the quantity $\mathcal{E}=\epsilon_{\infty} / \rho^{1 / 3}$ rather than $\epsilon_{\infty}$ itself.

The first thing to notice in Fig. 2 is that the wind zone, characterized by the central white region with $v \approx c$ and oblate shape, is smaller in run A, because of the stronger pinching forces around the equator. This difference in the size of the termination shock in the two cases will have visible consequences in the

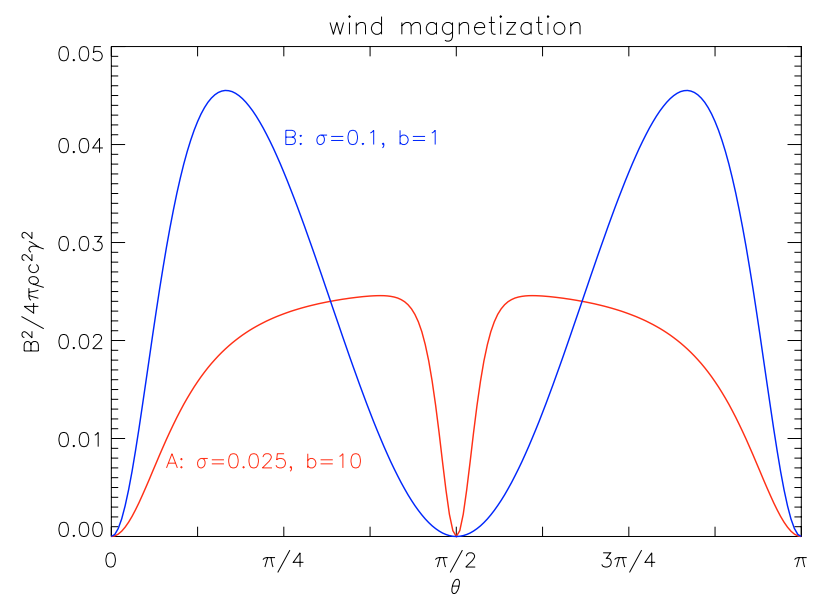

Fig. 1. The wind magnetization $B^{2} / 4 \pi \rho c^{2} \gamma^{2}$ as a function of the polar angle $\theta$, at any radius $r$, for the two runs defined in Eq. (37). In both cases the averaged value is approximately the same: $\sigma_{\text {eff }} \approx 0.02$.

emission maps. The flow structure is similar: in both cases substantial velocities are present in the equatorial plane as well as along the polar axis (the supersonic jets). It should be emphasized that the values we find, $v \approx 0.5-0.8 c$, are in agreement with those observed in the Crab Nebula (Hester et al. 2002) and in Vela (Pavlov et al. 2003). The complex flow pattern around the termination shock is also similar, but hoop stresses, which divert the flow toward the polar axis through small scale vortices, occur closer to the termination shock itself in case A, due to a much larger region of increased magnetization around the equator. This is clearly visible in the central panels: equipartition is reached or even exceeded in a large sector around the equator for run $\mathrm{A}$, while this is the case basically just along the termination shock front for run B, due to local compressions of the magnetic field by the flow vortices. Notice that the situation described here is very different from that encountered in standard stationary or self-similar radial models, where the nebular plasma reaches equipartition only at large radii. This is entirely due to the relaxation of the 1-D approximation, and to the complex flow pattern around the termination shock determined by the anisotropic wind energy flux.

The two panels on the right hand side show $\epsilon_{\infty}$, that is the highest possible energy of emitting particles at a given location. As we can see, in both cases the pattern resembles that of velocity, as could be expected given that the particle energy is advected by the flow. On the other hand, these rightmost panels are the "negative" of the corresponding central ones representing the magnetization structure. This is due to synchrotron losses. And again these losses determine the difference between the maps of $\epsilon_{\infty}$ for the two runs: while in case A the external parts of the map are dark (a lower cut at $\epsilon=10^{6}$ has been imposed), in run B the same regions have a higher maximum energy. This different behavior arises from the fact that in run A particles loose most of their energy in the high magnetization equatorial channel, whereas in case B, with a lower magnetization there, higher energy particles survive and are then transported across the nebula by the large scale vortices. The structure of the magnetic and velocity fields and the resulting distribution of $\epsilon_{\infty}$ will have important consequences, especially for the spectral properties of synchrotron emission, as will be widely discussed in Sect. 4.4. 

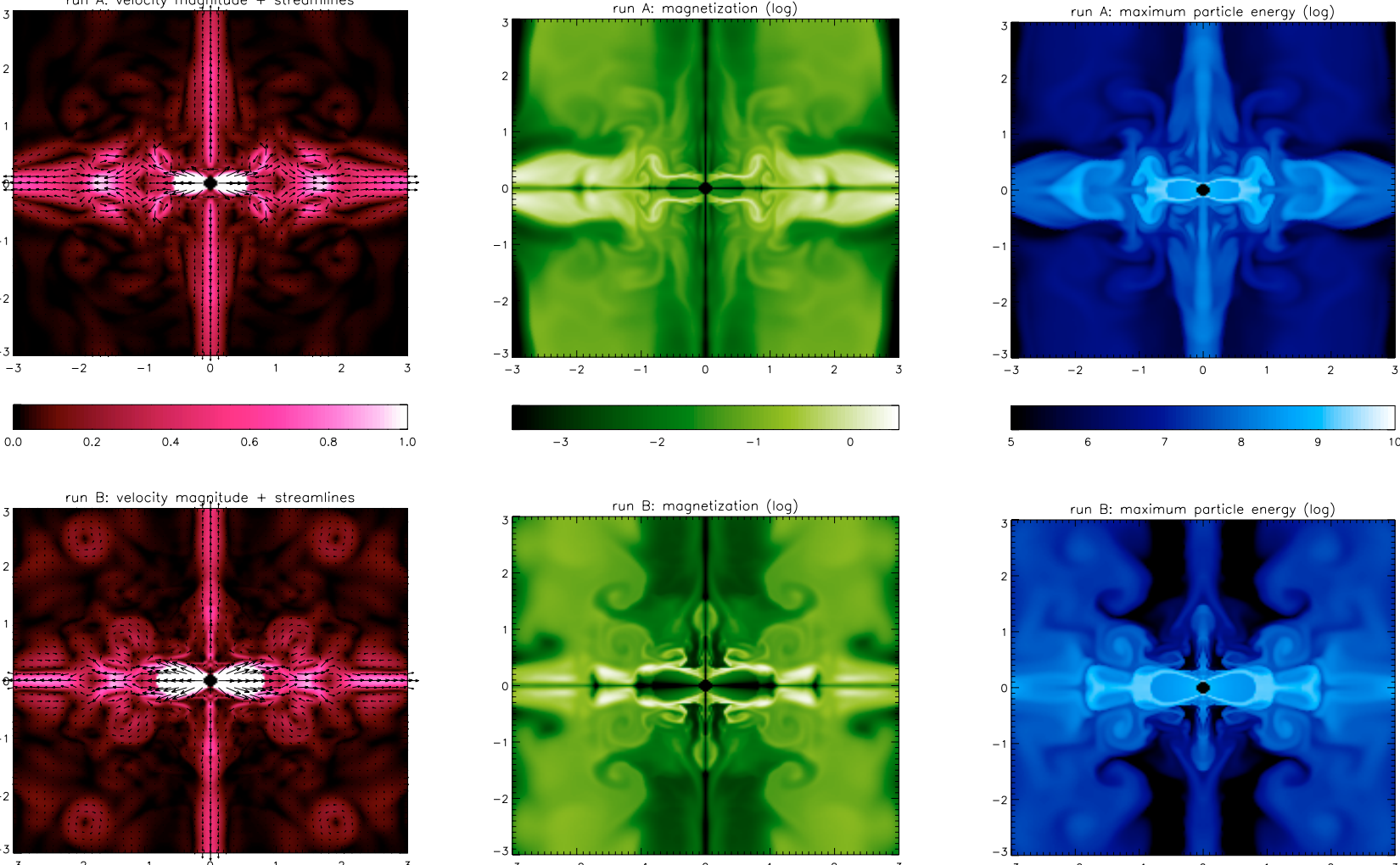

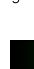
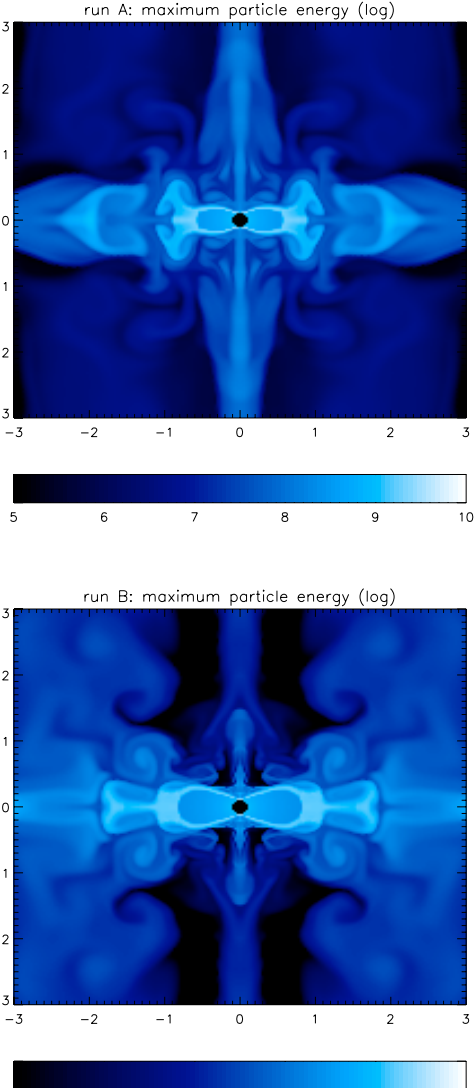

Fig. 2. Velocity magnitude (in units of $c$ ) and streamlines (left panels), magnetization (center panels), and maximum particle energy $\epsilon_{\infty}$ (in units of $m_{\mathrm{e}} c^{2}$, right panels) in the inner region of the simulated PWN, for run A (upper row) and run B (lower row). Distances from the central pulsar are reported on the axes, expresses in light year (ly) units.

\subsection{Surface brightness maps}

In order to obtain synchrotron emission maps, the spectral index $\alpha$ of the power law in Eq. (1) must be assigned. It is well known that in the case of the Crab Nebula more than a single power law at injection is needed in order to explain the observed spectrum from the radio to the X-ray band (e.g. Amato et al. 2000). Here we will assume that particles responsible for optical and X-ray emission come from the same distribution function, injected at the termination shock front, while the radio emitting particles are not considered. Therefore we take $\alpha=0.6$, in agreement with the values reported in optical spectral index maps (Véron-Cetty \& Woltjer 1993) close to the termination shock, where synchrotron burn-off has not occurred yet. Note that this value satisfies the condition $2 \alpha-1 \gtrsim 0$, needed to approximate the distribution function in Eq. (8) with the simple power law in Eq. (14). We have verified, by measuring the ratios $p / p_{0}$ and $p_{0} / n_{0}$, that the combined effect of the neglected terms leads to changes in the emissivity of $\sim 10 \%$, at most.

In Fig. 3 surface brightness maps are shown in optical and $\mathrm{X}$-rays for runs A and B (in logarithmic scale and normalized to the respective maximum value). Optical images are calculated for $\lambda=5364 \AA$ (left panels), one of the wavelengths selected by Véron-Cetty \& Woltjer (1993), at which emission from the outer filaments is negligible. For the X-rays, instead, we use $h v=1 \mathrm{keV}$, a value in the range of both the satellites Chandra and XMM-Newton. The images are obtained by applying Eq. (24) and assuming an inclination of the symmetry axis of $30^{\circ}$ with respect to the plane of the sky and of $48^{\circ}$ with respect to the North, values appropriate for the Crab Nebula (e.g.
Weisskopf 2000). A square box of 6 ly $\times 6$ ly, centered on the pulsar position, is used for our synthetic images.

The first thing to notice is that the emission is more distributed at optical frequencies compared with the X-rays, where the external regions appear darker. This is the expected effect of synchrotron burn-off, that causes the sources to appear smaller with increasing observation frequency. The central regions are instead rather similar in the two bands, with evidence for brighter features like rings, arcs and a central knot. The latter, in particular, tends to dominate the overall emission (especially in run B) if a linear scale is employed for the image. As it was first shown by Komissarov \& Lyubarsky (2004), these bright features are the result of Doppler boosted emission from fluid elements moving at relativistic speeds toward the observer. From a comparison with Fig. 2 it is clear that the regions with high velocity and magnetization, and thus higher emissivity, are those around the termination shock: the multiple rings observed are due to the external vortices produced by the hoop stresses which divert the flow, the brighter arc is due to flow along the termination shock, while the knot is due to the fluid escaping from the polar cusplike region toward the observer (see the above cited paper for a graphical explanation).

Morphological differences arise from the comparison of run $\mathrm{A}$ and $\mathrm{B}$, in the $\mathrm{X}$-ray maps especially, where the inner structure is highlighted. Run A is probably more reminiscent of the Crab Nebula: the external torus and the two polar jets are well visible, as well as the knot (first discovered by Hester et al. 1995, in optical Hubble Space Telescope images) and a system of wisps, with a brighter arc in what is usually called the inner 

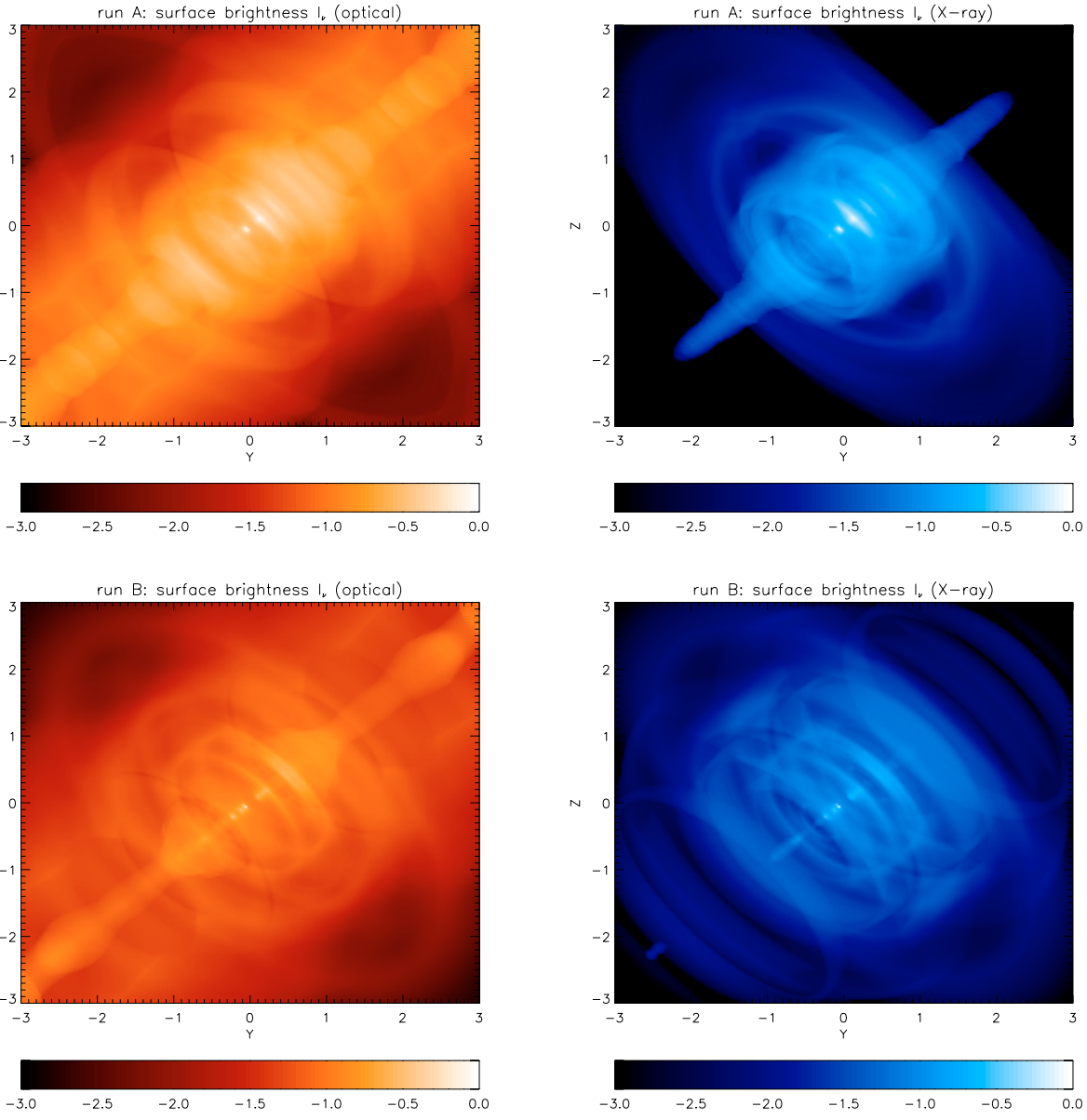

Fig. 3. Maps of surface brightness $I_{v}$, in logarithmic scale and normalized to the maximum value, for run A (upper row) and run B (lower row). Optical images $\left(5364 \AA\right.$ ) are displayed in left panels, X-ray images $(1 \mathrm{keV})$ on the right. The PWN axis of symmetry has been inclined by $30^{\circ}$ with respect with the plane of the sky and of $48^{\circ}$ with respect to the North, to compare with images of the Crab Nebula. For a distance $d \simeq 2 \mathrm{kpc}$, we have 1 ly $\simeq 32^{\prime \prime}$.

ring (Weisskopf et al. 2000). Notice that for this feature we find a radius of $0.18 \mathrm{pc}$, which is reasonably close to the observed value of $0.14 \mathrm{pc}$. The torus is associated with the higher magnetization equatorial region approximately between 1 and 3 ly from the pulsar (see again Fig. 2), in particular the emission is higher at $\sim 2$ ly, where the flow converges advecting emitting particles coming from higher latitudes (see the corresponding zone with higher values of $\epsilon_{\infty}$ ). A feature common to both structures is the fading of the emission toward the borders, far from the polar axis, where $\left|\boldsymbol{B}^{\prime} \times \boldsymbol{n}^{\prime}\right|$ becomes small in the coefficient of Eq. (18), due to the hypothesis of a purely toroidal field (see the discussion below).

The jets are hollow, since the toroidal field vanishes on the axis, but in run A their emission is significant because emitting particles are efficiently transported there by the high speed flow. This last effect is reduced in run B, where the X-ray jets basically disappear, while the diffuse X-ray emission is enhanced in the nebula. The explanation is again found by looking at Fig. 2: in run B, the hoop stresses are less efficient, due to the wider low magnetization region, and particles lose more energy through synchrotron radiation before flowing toward the axis. It is important to notice that, in both cases, jets are visible in the optical band, and actually, at lower frequencies, the difference between their appearance in the two runs is reduced. However they are not so bright now with respect to their surroundings and the diffuse emission from the outer nebula hides them, precisely as it happens in the observations. In the central region, other than the (overly) bright knot, two boosted arcs are visible in run B, reminiscent of the situation encountered in the Vela PWN (Helfand et al. 2001; Pavlov et al. 2001).

Summarizing, it appears that run A, which has a more magnetized wind near the equator, leads to a brighter X-ray torus and to a single inner Doppler boosted arc, whereas run B, where the low magnetization region around the equator is more extended, implies a more diffuse nebular emission and a more complex system of rings and arcs in the central region.

Before concluding this sub-section, let us comment on the consequences of the assumption of a purely toroidal magnetic field. Although evaluation of the dynamical effects of a poloidal field component would require a full 3-D treatment, that is beyond our present goal, the effects of an isotropic field on the emission properties are straightforward to check. If we thus substitute the term $\left|\boldsymbol{B}^{\prime} \times \boldsymbol{n}^{\prime}\right|$ in the emissivity with its average over the solid angle (compare with Eq. (23)):

$B_{\perp}^{\prime}=\left|\boldsymbol{B}^{\prime} \times \boldsymbol{n}^{\prime}\right|=\sqrt{\frac{2}{3}} \frac{B}{\gamma}$,

we obtain the results shown in Fig. 4, where only case A has been considered (similar differences arise for case B). The main difference, at a given frequency, between the corresponding images in Figs. 3 and 4 is the relative brightness of some of the small scale features mentioned above. First of all the knot 

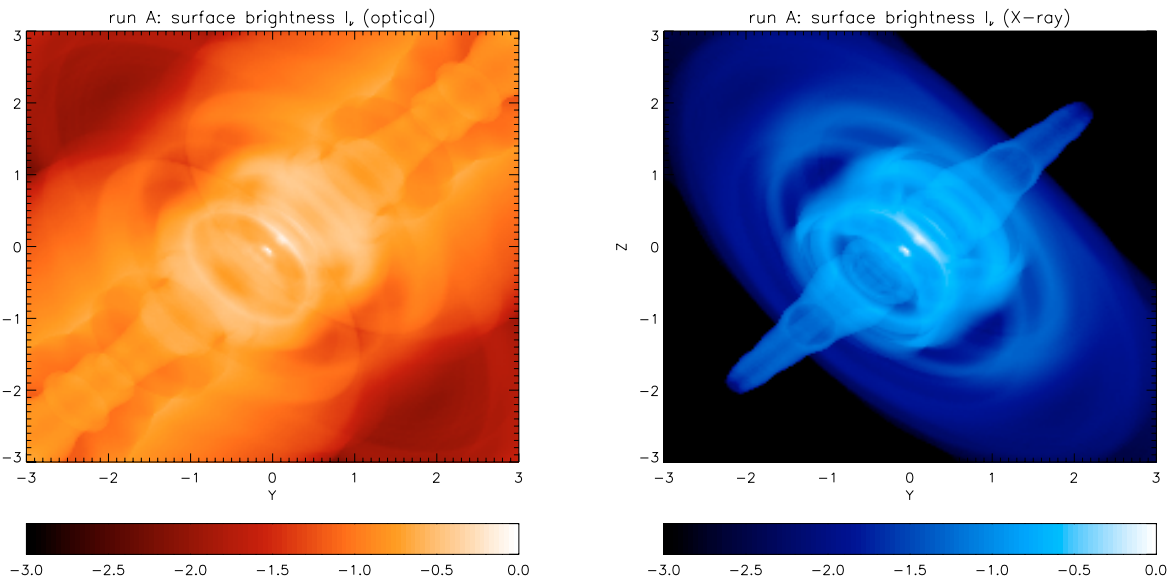

Fig. 4. Maps of surface brightness for run A corresponding to those in Fig. 3 obtained with an isotropic magnetic field.
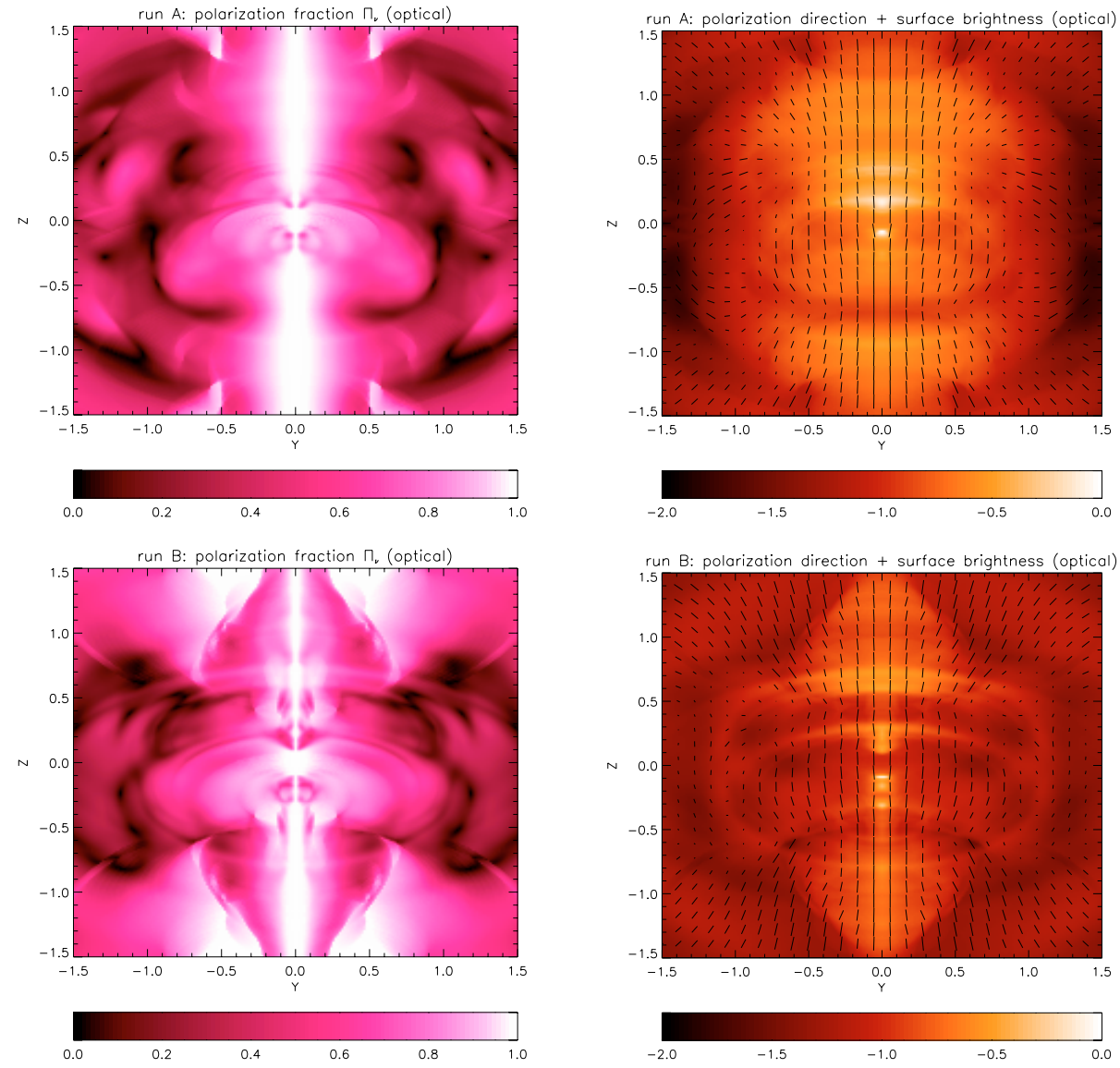

Fig. 5. Maps of (optical) polarization fraction $\Pi_{v}$ (left) and direction (right, superimposed to the surface brightness map), for run A (upper row) and run B (lower row). Polarization fraction is normalized against $\frac{\alpha+1}{\alpha+5 / 3} \simeq 70 \%$, and the ticks length used for polarization direction is proportional to the normalized fraction.

appears to be less dominant than before, as well as the main arc in the inner ring. Moreover, as one would expect, the outer parts of all ring-like structures are enhanced. In particular, looking at the X-ray map, we notice that what we here have referred to as the inner ring is now more uniform, as in the observations (Weisskopf et al. 2000).

\subsection{Polarization maps}

Let us now concentrate on the (linear) polarization properties of the nebular emission. A preliminary study was reported in an earlier paper (Bucciantini et al. 2005b), where maps of the polarization fraction $\left(\Pi_{v}\right)$ and direction based on a similar simulation were presented. In Fig. 5 we show the same maps for runs A and $\mathrm{B}$, where the central region is zoomed and not rotated in the plane of the sky for ease of interpretation (the tilt is retained). The two cases are similar: the polarized fraction is high along the polar axis, where the projected magnetic field is always orthogonal to the line of sight, while depolarization occurs in the outer regions, where contributions from projected fields with opposite signs sum up along the line of sight. On the right hand side we show the polarization direction, with ticks proportional to the fraction displayed on the left, together with the corresponding 

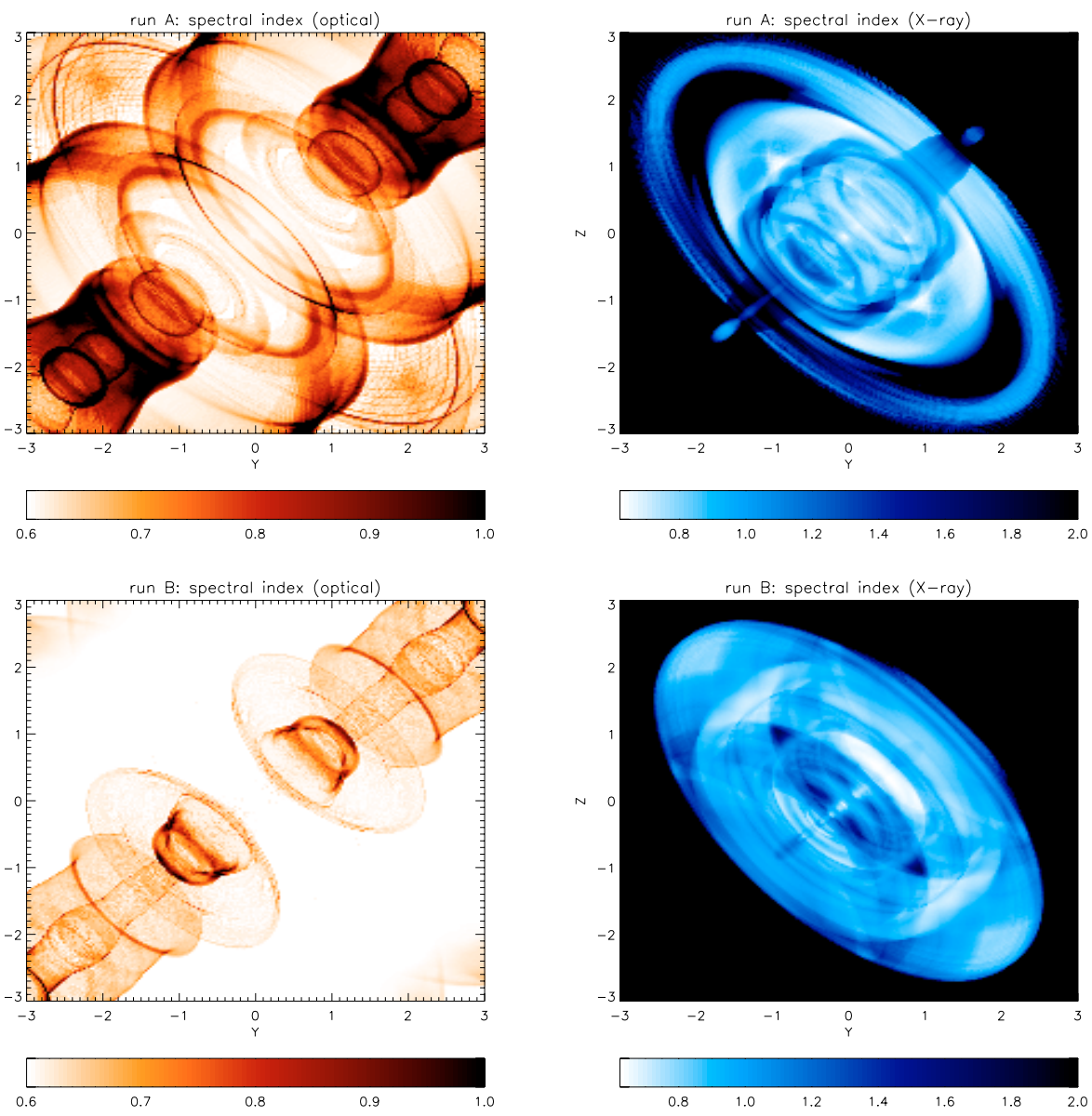

Fig. 6. Maps of spectral index $\alpha_{v}$, as defined in Eq. (32), for run A (upper row) and run B (lower row). Optical images are obtained by comparing specific intensities at $\lambda=5364 \AA$ and $\lambda=9241 \AA$ and are displayed in left panels. X-ray images are obtained by using energies of 0.5 and 8 keV, and are displayed on the right.

map of surface brightness, with the aim of making clear the association between the polarization behaviour and the emission main features. As we can see, polarization ticks are basically always orthogonal to the toroidal field, displaying the behaviour expected given the inclination of the symmetry axis with respect to the plane of the sky. However, in the rings where the velocity is relativistic, deviations of the vector direction due to polarization angle swing are also visible (e.g. in the arc of the torus, that is the second brightest arc above the central knot).

Concerning differences between the two runs, we notice that the structure of polarization fraction and direction becomes more complex for run $\mathrm{B}$, for which also the magnetization map in Fig. 2 was more complicated, leading to the presence of multiple rings. Notice also that in these zoomed maps it is possible to see a small displacement (to the South) of the knot with respect to the central position, as in the figures by Hester et al. (1995). Unfortunately, high resolution optical polarization maps of the inner region of the Crab Nebula are not available yet: all papers mentioned in the Introduction refer to the whole nebula, where contributions from the confining medium may be significant. Finally, for X-ray polarimetry, which could really provide crucial clues to the magnetic structure in the inner region, the necessary technology is still to come.

\subsection{Spectral index maps and integrated spectra}

Consider now the spectral properties, starting with the maps of spectral index $\alpha_{v}$ in Fig. 6, both in the optical and X-ray bands.
Optical images are obtained by comparing specific intensities at $\lambda=5364 \AA$ and $\lambda=9241 \AA$, wavelengths that have been chosen so as to allow direct comparison with the observational data presented in the paper by Véron-Cetty \& Woltjer (1993). The X-ray spectral index maps, instead, are obtained by using energies of 0.5 and $8 \mathrm{keV}$, as in Mori et al. (2004).

The optical images do not show significant variations from the injection value of $\alpha=0.6$ in the inner region. Once again run $\mathrm{A}$ seems to be more closely representative of the situation in the Crab Nebula, with spectral softening occurring, starting from a distance of about $0.5 \mathrm{pc}$ from the central pulsar position, in the vicinity of the polar axis, and more gradually at larger cylindrical radii. This is consistent with observations, at least qualitatively, since softening, in the optical band, is present only beyond the torus. A general consideration is that in our runs, and especially in run B, the nebular magnetic field in the central region seems to be weaker than the value usually assumed for the average field in the Crab Nebula $\sim 3 \times 10^{-4} \mathrm{G}$. In the torus, our simulations give a field strength lower by a factor of 2 , for run $\mathrm{A}$, and 3 , for run $\mathrm{B}$. In addition to that, when comparing to real images, one should also consider that dimensions must be rescaled somehow. While in run A the positions of the main features are roughly in agreement with the observations (see the discussion in Sect. 4.2), in run B the radii of the inner ring and the torus are approximately two times larger than observed. This naturally concurs to the spectral flatness of the corresponding map. 


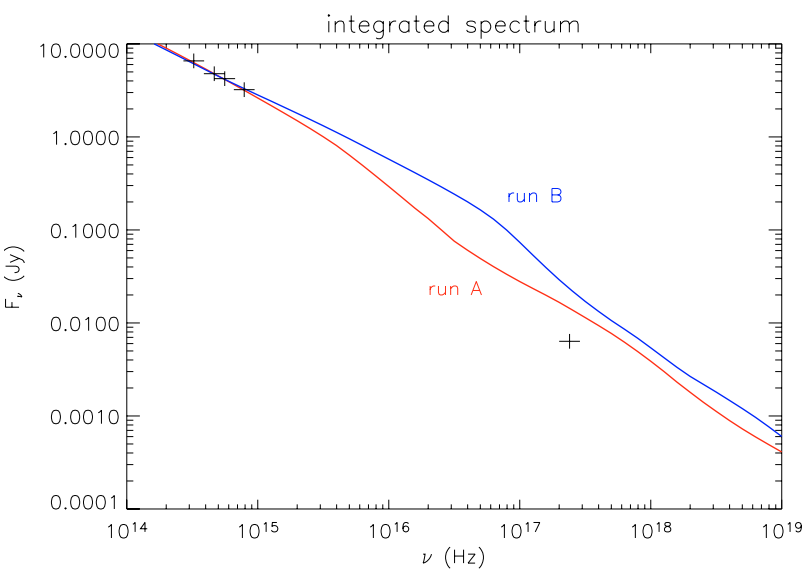

Fig. 7. The integrated spectrum as a function of frequency for the two runs. Spectra are normalized to $\lambda=5364 \AA$.

In the X-rays synchrotron burn-off occurs on much shorter distances and the maps are much more shaped: spectral softening is much stronger and, as a consequence, basically only the inner structure is visible in the adopted range. Notice how the values are similar to those measured by Mori et al. (2004) (where photon index is used for the scale, that is $\alpha_{v}+1$ ), and in run $\mathrm{A}$ we even observe the brighter (harder) features in the polar jets, especially the southern one.

Finally, let us calculate the integrated spectra (net fluxes) as in Eq. (33), for the two runs A and B, and compare them with spectro-photometric data of the Crab Nebula, at optical and X-ray frequencies. In Fig. 7 the net flux $F_{v}$ (in Jansky) is plotted against frequency $v$ (in Hertz). Solid lines are the synthetic integrated spectra obtained from simulations. The four crosses in the optical range correspond to data at $\lambda=9241,6450,5364$, and $3808 \AA$, corrected for interstellar absorption and thermal contribution from the filaments (Véron-Cetty \& Woltjer 1993), whereas the $\mathrm{X}$-ray data point at $1 \mathrm{keV}$ is taken from Willingale et al. (2001) and refers to XMM-Newton observations. We can notice the different behaviour of the two runs. For run $\mathrm{A}$ the spectrum steepens because of synchrotron burn-off already in the optical band, where the slope is 0.75 rather than the injection value of 0.6. A spectral break is seen at $v_{\mathrm{b} 1} \approx 2-3 \times 10^{15} \mathrm{~Hz}$, as expected (e.g. Véron-Cetty \& Woltjer 1993), with the slope changing to 1.15 , yielding an increase of 0.55 , comparable to the value 0.5 usually adopted. On the other hand, in run $B$, the lower magnetic field causes losses to be negligible in the optical and the break to move toward X-ray photon energies.

The most puzzling feature is the presence in both cases of further, unexpected, changes of slope, with the appearance of what we term as inverse breaks, i.e. flattenings of the spectrum. Focusing on the results relative to run $\mathrm{A}$, we see that the spectral index has a second change from 1.15 to 0.8 at around $v_{\mathrm{b} 2} \sim$ $3 \times 10^{16} \mathrm{~Hz}$; then it increases again to 1.1 at $\nu_{\mathrm{b} 3} \sim 10^{18} \mathrm{~Hz}$; and finally decreases to 0.85 at $v_{\mathrm{b} 4} \sim 3 \times 10^{18} \mathrm{~Hz}$. The behavior of run $\mathrm{B}$ is qualitatively analogous, although there are differences in the position and entity of the changes of slope.

The reason for this behavior is better understood by looking at Fig. 8 where we show the cut-off frequency for run $\mathrm{A}$ as a function of space. In particular, if one looks at the contours, it is clear that while the initial break at $\nu_{\mathrm{b} 1}$ is due to loss of emission from high latitudes, that particles with energies corresponding to emission at $v>v_{\mathrm{b} 1}$ are not able to reach (consider the contours referring to $10^{15}$, solid line, and $10^{16} \mathrm{~Hz}$, dotted line, in the figure), the inverse break at $v_{\mathrm{b} 2}$ appears because there is a range of
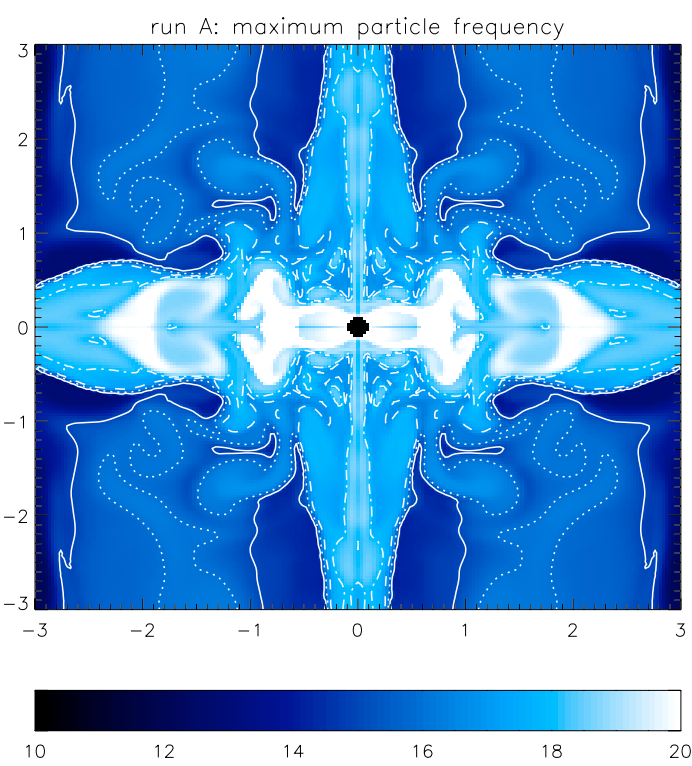

Fig. 8. The distribution of the maximum particle frequency (in $\mathrm{Hz}$ units), that is the critical frequency of Eq. (19) calculated ignoring line of sight effects. Contours refer to the values of $10^{15} \mathrm{~Hz}$ (solid line), $10^{16} \mathrm{~Hz}$ (dotted line), $10^{17} \mathrm{~Hz}$ (dashed line), $10^{18} \mathrm{~Hz}$ (dot-dashed line).

frequencies for which the emitting region keeps constant in size. This is strictly related to the particular configuration of the magnetic field (see the magnetization map in Fig. 2) that is mainly confined to the equatorial region due to the presence of large scale vortices. This is confirmed by the spatial ditribution of the maximum particle energy $\epsilon_{\infty}$, which for high frequencies (thus in X-rays) appears to be concentrated just around the termination shock, so that a further increase in frequency does not lead to any further reduction of the emitting region.

The result of the deviations from the expected monotonic spectral steepening described above is that X-ray emission is too high, by a factor of 2 in run $\mathrm{A}$ and 3 in run $\mathrm{B}$. This difference of values between the two runs mostly depends on the different strength of the magnetic field which causes stronger synchrotron losses for run A than for run B, although still not enough to explain the observations quantitatively.

\section{Conclusions}

In this paper a complete suite of diagnostic tools for deriving synchrotron emission properties from numerical relativistic MHD simulations has been presented. The method is quite simple and general, so that it can be applied to any numerical scheme and to any astrophysical source of synchrotron radiation. The application presented here concerns the emission from PWNe, based on the axisymmetric model by Del Zanna et al. (2004). In this case, synchrotron radiation is the primary diagnostic for the physics in the source and may give important clues about the structure of the pulsar wind itself, since its properties are found to be reflected in the nebular emission. In particular we have studied the differences arising from two different magnetic structures of the wind: one in which the striped (low magnetization) wind region is very narrow, and the toroidal field has a maximum near the equator (run A), and another where the maximum occurs at a much higher latitude above the equator (run B). In both runs the average magnetization was the same, $\sigma_{\text {eff }} \approx 0.02$, and high enough to induce the presence of supersonic polar jets. 
The width of the striped wind region of low magnetization is usually considered as directly linked to the inclination angle between the pulsar's rotation and magnetospheric axes, thus such a study could yield crucial information otherwise difficult to obtain. By looking at surface brightness maps, especially in X-ray, it appears that run A produces images which are remarkably similar to that of the Crab Nebula observed by the ROSAT (Hester et al. 1995), Chandra (Weisskopf et al. 2000), and XMM-Newton (Willingale et al. 2001) satellites. An equatorial torus and two polar jets are clearly visible, together with an inner ring, with Doppler boosted emission concentrated in a main arc, and a bright knot just SE from the pulsar position. Particularly noticeable is the presence of the jets, necessarily hollow because of our assumption of axisymmetry and of a purely toroidal field (though the case of an isotropic field has been treated here too): these did not appear as emitting features in previous simulations. Run B produces instead maps which resemble vaguely the Vela PWN (Helfand et al. 2001; Pavlov et al. 2001, 2003), with a couple of bright arcs NW from the central knot in the inner region. This is entirely due to the different flow structure determined by the lower magnetization around the equator (hoop stresses and small scale vortices develop at higher latitudes).

In the present paper we have used observations mainly of the Crab Nebula as a benchmark for our diagnostic techniques, for the simple reason that the Crab Nebula is by far the best studied PWN. We have briefly mentioned the Vela case too, but one may wonder whether by tuning our model parameters the resulting surface brighness maps could reproduce also different features encountered there or in some other PWN. One of the most puzzling problems has always been the jet in the Vela PWN, which is much brigher on the wrong side of the pulsar position (that for which the jet is receding from us). The best explanation is probably to invoke kink-type instabilities that manage to bend sections of the counter-jet toward us, thus enhancing their emission, but in order to test this idea full 3-D simulations would be required. Regarding other objects, we may consider the case of the PWN around pulsar B1509-58. In the central region multiple knots are observed (Gaensler et al. 2002) and the brightest ones are located between the pulsar position and the boosted arc on the torus, opposite to the direction of the (main) jet. Again, these features cannot be explained within our 2-D model. Possibly, they could arise due to time dependent small-scale Kelvin-Helmholtz instabilities, as it has been suggested for the variable optical wisps and sprites in the Crab Nebula (Begelman 1999; Bucciantini \& Del Zanna 2006), but in any case the approximation of axisymmetry must be relaxed. In the same source, the ring-like region named RCW 89 could be produced by the counter-jet (very faint in the Chandra images) hitting the contact discontinuity between the PWN and the expanding ejecta. This scenario is well within our assumptions and its accurate modeling is left as future work.

We have also computed detailed polarization maps. This is expected to be a very powerful diagnostic technique for the magnetic structure of the inner regions of PWNe, since synthetic surface brightness maps are likely to be too largely dominated by Doppler boosted features, in the vicinities of the central source, to be sensitive to small changes in the magnetic field strength and direction (see Bucciantini et al. 2005b, for a wider discussion). The results show a high degree of linear polarization close to the axis of symmetry (not far from the theoretical maximum of $70 \%$, given the spectral index $\alpha=0.6$ ), and a strong depolarization in the outer equatorial regions. Relativistic effects related to Doppler boosting (polarization angle swing) are also visible in the brighter arcs. High resolution optical, not to mention X-rays, polarization observations, needed for a comparison of the numerical results with the real data, aimed at probing the inner PWN magnetic structure, are lacking, at present, but are highly desirable given their diagnostic potential.

Synthetic spectral index maps have been computed here for the first time on the basis of MHD numerical simulations. At a qualitative level, the results of our run A are in good agreement with both optical (Véron-Cetty \& Woltjer 1993) and X-ray (Mori et al. 2004) data: spectral softening is weak in the central region and stronger at the borders of the torus, especially in the $\mathrm{X}$-rays, where even the harder features inside the jets highlighted in Chandra data are reproduced. However, the nebular field appears to be too low to explain the observations quantitatively, giving too weak a softening, especially for run $\mathrm{B}$. This is further confirmed when looking at the integrated spectra, which show that the spectral break is too weak and occurs at a too high frequency. The result is that, if we normalize the spectra to a flux in the optical, X-ray data are over-estimated by a factor 2 for run A and 3 for run $\mathrm{B}$. We have attributed this behavior to the distribution of the nebular magnetic field and, consequently, to that of the maximum energy of emitting particles which serves as a local synchrotron cut-off. Such distributions basically show that the magnetic field is too strongly affected by the compression due to large scale flow vortices and piles up around the termination shock. We have also verified that this situation invariably occurs for different values of the simulation parameters.

In spite of these successes in PWN modeling, in order to proceed further 3-D simulations are certainly required. The strongest limitation appears to be the assumption of a purely toroidal magnetic field, both for the overall dynamics (e.g. allowing for kink-type instabilities along the axis and possibly reconnection at the equator) and for the emission properties, where a certain amount of disordered magnetic field on small scales could help to reproduce the observations, as we have shown in maps for the optical and X-ray bands in the case of an isotropic field (see also the discussion in Shibata et al. 2003). Another issue not properly addressed here is time variability: though the overall evolution of the PWN/SNR system is nearly self-similar in the free expansion phase, the small scale vortices around the termination shock are variable on a time scale of a few years (see also Bogovalov et al. 2005). We leave the discussion of these two important issues to future papers.

Acknowledgements. The authors thank R. Bandiera, F. Pacini, J. Arons, S. Shibata, and S. Komissarov for valuable discussions, and the referee P. Slane for helping us to improve the paper. This work was partly supported by MIUR under grant Cofin 2004 (Pacini).

\section{References}

Amato, E., Salvati, M., Bandiera, R., Pacini, F., \& Woltjer, L. 2000, A\&A, 359, 1107

Antón, L., Zanotti, O., Miralles, J. A., et al. 2006, ApJ, 637, 296

Arons, J. 2004, Adv. Sp. Res., 33, 466

Begelman, M. C. 1999, ApJ, 512, 755

Begelman, M. C., \& Li, Z.-Y. 1992, ApJ, 397, 187

Björnsson, C.-I. 1982, ApJ, 260, 855

Blandford, R. D., \& Königl, A. 1979, ApJ, 232, 34

Bogovalov, S. V., \& Khangoulian, D. V. 2002, MNRAS, 336, L53

Bogovalov, S. V., Chechetkin, V. M., Koldoba, A. V., \& Ustyugova, G. V. 2005, MNRAS, 358, 705

Bucciantini, N., \& Del Zanna, L. 2006, A\&A, in press [arXiv: astro-ph/0603481]

Bucciantini, N., Blondin, J. M., Del Zanna, L., \& Amato, E. 2003, A\&A, 405, 617

Bucciantini, N., Amato, E., \& Del Zanna, L. 2005a, A\&A, 434, 189

Bucciantini, N., Del Zanna, L., Amato, E., \& Volpi, D. 2005b, A\&A, 443, 519

Camilo, F., Gaensler, B. M., Gotthelf, E. V., Halpern, J. P., \& Manchester, R. N. 2004, ApJ, 616, 1118 
Coroniti, F. V. 1990, ApJ, 349, 538

Del Zanna, L., \& Bucciantini, N. 2002, A\&A, 390, 1177

Del Zanna, L., Bucciantini, N., \& Londrillo, P. 2003, A\&A, 400, 397

Del Zanna, L., Amato, E., \& Bucciantini, N. 2004, A\&A, 421, 1063

Duez, M. D., Liu, Y. T., Shapiro, S. L., \& Stephens, B. C. 2005, Phys. Rev. D, 72,4028

Emmering, R. T., \& Chevalier, R. A. 1987, ApJ, 321, 334

Font, J. A. 2003, Living Rev. Relativity, 4

Gaensler, B. M., Pivovaroff, M. J., \& Garmire, G. P. 2001, ApJ, 556, 380

Gaensler, B. M., Arons, J., Kaspi, V. M., et al. 2002, ApJ, 569, 878

Gammie, C. F., McKinney, J. C., \& Tóth, G. 2003, ApJ, 589, 444

Gotthelf, E. V., \& Wang 2000, ApJ, 532, L117

Helfand, D. J., Gotthelf, E. V., \& Halpern, J. P. 2001, ApJ, 556, 107

Hester, J. J., Scowen, P. A., Sankrit, R., et al. 1995, ApJ, 448, 240

Hester, J. J., Mori, K., Burrows, D., et al. 2002, ApJ, 577, L49

Hickson, P., \& van der Bergh, S. 1990, ApJ, 365, 224

Kennel, C. F., \& Coroniti, F. V. 1984a, ApJ, 283, 694

Kennel, C. F., \& Coroniti, F. V. 1984b, ApJ, 283, 710

Komissarov, S. S. 1999, MNRAS, 303, 343

Komissarov, S. S., \& Lyubarsky, Y. E. 2003, MNRAS, 344, L93

Komissarov, S. S., \& Lyubarsky, Y. E. 2004, MNRAS, 349, 779

Komissarov, S. S. 2005, MNRAS, 359, 801

Londrillo, P., \& Del Zanna, L. 2004, J. Comput. Phys., 195, 17

Lyubarsky, Y. E. 2002, MNRAS, 329, L34

Lyubarsky, Y. E., \& Eichler, D. 2001, ApJ, 562, 494

Lyutikov, M., Pariev, V. I., \& Blandford, R. D. 2003, ApJ, 597, 998
Lu, F. J., Wang, Q. D., Aschenbach, B., Durouchoux, P., \& Song, L. M. 2002, ApJ, 568, L49

Martí, J. M., \& Müller, E. 2003, Living Rev. Relativity, 6

Michel, F. C. 1973, ApJ, 180, 133

Michel, F. C., Scowen, P. A., Dufour, R. J., \& Hester, J. J. 1991, ApJ, 368, 436

Mori, K., Burrows, D. N., Hester, J. J., et al. 2004, ApJ, 609, 186

Pavlov, G. G., Kargaltsev, O. Y., Sanwal, D., \& Garmire, G. P. 2001, ApJ, 554, L189

Pavlov, G. G., Teter, M. A., Kargaltsev, O. Y., \& Sanwal, D. 2003, ApJ, 591, 1157

Romani, R. W., Ng, C.-Y., Dodson, R., \& Brisken, W. 2005, ApJ, 631, 480

Rybicki, G. B., \& Lightman, A. P. 1979, Radiative Processes in Astrophysics (New York: Wiley)

Schmidt, G. D., Angel, J. R. P., \& Beaver, E. A. 1979, ApJ, 227, 106

Shibata, S., Tomatsuri, H., Shimanuki, M., Saito, K., \& Mori, K. 2003, MNRAS, 346,841

Shklovsky, I. S. 1953, Dokl. Akad. Nauk SSSR, 90, 983

Slane, P., Helfand, D. J., van der Swaluw, E., \& Murray, S. S. 2004, ApJ, 616, 403

van der Swaluw, E., Achterberg, A., Gallant, Y. A., \& Tóth 2001, A\&A, 380, 309

Velusamy, T. 1985, MNRAS, 212, 359

Véron-Cetty, M. P., \& Woltjer, L. 1993, A\&A, 270, 370

Weisskopf, M. C., Hester, J. J., Tennant, A. F., et al. 2000, ApJ, 536, L81

Willingale, R., Aschenbach, B., Griffiths, R. G., et al. 2001, A\&A, 365, L212

Wilson, A. S. 1972, MNRAS, 157, 229 